\title{
ABSTRACT CAUCHY PROBLEMS OF THE ELLIPTIC TYPE
}

BY A. V. BALAKRISHNAN

Communicated by Einar Hille, June 13, 1958

Let $A$ be the infinitesimal generator of a strongly continuous oneparameter semigroup $T(\xi), 0<\xi$, of endomorphisms over a $B$-space $X$. Suppose it is required to find a function $u(t), 0<t$, with values in $X$ such that:

(i) $u(t), u^{1}(t), \cdots, u^{n-1}(t)$ are absolutely continuous, $u^{k}(t)$ being the derivative of $u^{k-1}(t)$.

(ii) $u^{n}(t)=(-1)^{n+1} A u(t)$.

(iii) $\left\|u^{k}(t)-u_{k}\right\| \rightarrow 0$, as $t \rightarrow 0+, k=0, \cdots, n-1$.

We call this an abstract Cauchy problem of the elliptic type $\left(\mathrm{ACPE}_{n}\right)$. We prove:

TheOREM 1. The $A C P E_{n}$ has at most one solution provided

$$
\int_{1}^{\infty}\|T(\xi)\| \xi^{-\sigma-1} d \xi<\infty \text { for every } \sigma>0
$$

ThEOREM 2. Let $n=2$. Let the semi-group $T(\xi)$ satisfy $\mathrm{H}_{1}$ and let $u(t)$ be any solution of the $A C P E_{2}$ such that

$$
\limsup _{t \rightarrow \infty} t^{-1} \log \|u(t)\| \leqq 0 .
$$

Then necessarily

$$
u(t)=\left(t / 2 \pi^{1 / 2}\right) \int_{0}^{\infty} T(\xi) u_{0} \xi^{-3 / 2} \exp \left(-t^{2} / 4 \xi\right) d \xi .
$$

A slightly different but useful version of Theorem 2 is:

Theorem 3. Let $n=2$. Let the semi-group $T(\xi)$ satisfy $\mathrm{H}_{1}$. Let $u(t)$, $t>0$, satisfy (i), (ii), but (iiia) below in place of (iii)

$$
\left\|u(t)-u_{0}\right\| \rightarrow 0 \text { as } t \rightarrow 0+\text {. }
$$

Then, if $u(t)$ satisfies $\mathrm{H}_{2}$ in addition, $u(t)$ is again determined by (1). Moreover, if $\|T(\xi)\| \rightarrow 0$ as $\xi \rightarrow \infty$, then any such $u(t)$ has a similar property, viz.:

$$
\|u(t)\| \rightarrow 0 \text {, as } t \rightarrow \infty .
$$

Results similar in principle have been obtained for other values of $n$. 
As an application of these results we may consider the elliptic equation:

$$
\frac{\partial^{2}}{\partial t^{2}} u(t, x)+a(x) \frac{\partial^{2}}{\partial x^{2}} u(t, x)+b(x) \frac{\partial}{\partial x} u(t, x)=0
$$

where the functions $u(t, \cdot)$ are to lie in $C[\alpha, \beta],-\infty \leqq \alpha<\beta \leqq \infty$, $a(x), b(x)$ are continuous and $a(x)>0$. It suffices to consider the Fokker-Planck equation

$$
\frac{\partial}{\partial t} u(t, x)=a(x) \frac{\partial^{2}}{\partial x^{2}} u(t, x)+b(x) \frac{\partial}{\partial x} u(t, x) .
$$

However, lateral conditions for semigroup solutions of (3) have been given by Feller and Hille. Theorem 3 thus yields, in particular, a corresponding result on the global boundedness of the solutions of (2) (the generalized Phragmén-Lindelöf principle of P. Lax) somewhat more general than obtained hitherto in that conditions on $a(x)$ and $b(x)$ are milder, being enough to insure semigroup solutions of (3).

University of Southern California 